\title{
A search for extremely high energy neutrinos in 6 years (2008-2014) of IceCube data
}

\author{
The IceCube Collaboration ${ }^{1}$ \\ ${ }^{1}$ http://icecube.wisc.edu/collaboration/authors/icrc15_icecube \\ E-mail: aya@hepburn.s.chiba-u.ac.jp
}

\begin{abstract}
Observations of extremely high energy neutrinos $\left(\gtrsim 10^{16} \mathrm{eV}\right)$ are expected to probe the origin of the highest energy cosmic rays with energies above $10^{20} \mathrm{eV}$. Cosmogenic neutrinos are produced in the interactions of those most energetic cosmic rays with cosmic microwave background photons (GZK effect) and considered a guaranteed astrophysical neutrino signal. The cosmogenic neutrinos have been searched using the partially and fully completed IceCube detector. We present the updated analysis of the extremely high energy neutrinos above $\sim 10^{6} \mathrm{GeV}$ in the total of 6 years of IceCube sample with 3 years of partially completed IceCube data taken in 2008-2011 and 3 years of completed IceCube data in 2011-2014. The sensitivity for a diffuse flux of cosmic neutrinos with an $E^{-2}$ spectrum in the central $90 \%$ energy range $300 \mathrm{TeV}$ to $2 \mathrm{EeV}$ is expected to be at a level of $E^{2} \phi \leq 3 \times 10^{-9} \mathrm{GeVcm}^{-2} \mathrm{sec}^{-1} \mathrm{sr}^{-1}$ and the improvement corresponds to more than a factor of 2.5 from the previous study with two year data from the nearly completed IceCube detector. The current analysis leads to further constrain or prove the highest energy cosmic-ray origin with the IceCube neutrino observatory.
\end{abstract}

Corresponding author: Aya Ishihara ${ }^{2 *}$

${ }^{2}$ Department of Physics, Graduate School of Science, Chiba University, Chiba 263-8522, Japan

The 34th International Cosmic Ray Conference,

30 July- 6 August, 2015

The Hague, The Netherlands

${ }^{*}$ Speaker. 


\section{Introduction}

Astrophysical neutrinos are expected to be produced by interactions of ultra-high-energy cosmicrays (UHECRs) with the surrounding photons and/or matter. These neutrinos, which are undeflected in the Galactic and extra-galactic magnetic field and unattenuated in the photon filled Universe, play an important role as information carriers of hard to identify cosmic accelerators. Moreover, cosmogenic neutrinos [1], also known as GZK neutrinos, that are induced by the highest energy cosmic-ray interactions with background photons in the Universe [2] are expected in the energy region above $\sim 10 \mathrm{PeV}$. These cosmogenic neutrinos are one of the most promising messengers from the high-energy, distant universe beyond $\mathrm{PeV}$ energies. They may provide us with direct evidence of the highest energy cosmic-ray sources. However, since the expected flux level of GZK neutrinos is so low, it requires a very large scale neutrino detector. IceCube is a cubic kilometer scale deep underground Cerenkov neutrino detector at the South Pole. The IceCube detector construction was completed in December 2010. The IceCube array comprises 5160 optical sensors on 86 cables, called strings, over a $1 \mathrm{~km}^{3}$ instrumented volume of ice at a depth of $1450 \mathrm{~m}$ to $2450 \mathrm{~m}$. Additional optical sensors frozen into tanks located at the surface near the top of each hole constitute an air shower array called IceTop. In 2008-2009, 2009-2010 and 2010-2011, 40, 59 or 79 out of 86 cables were deployed and taking data with an approximate fiducial volume of $0.5,0.7$ and $0.9 \mathrm{~km}^{3}$, respectively. Then in 2011-2014, IceCube accumulated data with the fully completed array. The previous search for cosmogenic neutrinos was carried out with 2 years of data, in which first year data was obtained with 79 strings and second year data was taken with the full array. While two PeV events were discovered by the search [3], no cosmogenic neutrinos were observed [4] and stringent limits were placed on cosmogenic neutrino model fluxes. It was shown that astrophysical objects with populations following a strong cosmological evolution, such as Fanaroff-Riley type II radio galaxies, are unlikely the highest energy cosmic-ray sources. In this proceedings, we present the expected sensitivity of the analysis in search for neutrinos above a PeV with the 6 years of IceCube data taken between April 2008 and May 2014 with an effective livetime of 2050 days.

\section{Cosmogenic neutrino events in IceCube}

Cosmogenic neutrino fluxes are, to the first order approximation, characterized by broken power-laws above a few PeV. A hard power-law spectrum with spectral index of $\sim-0.7$ is expected in the energy region below $100 \mathrm{PeV}$ as illustrated in Fig.1. This feature is robust against different highest energy cosmic-ray source models, as this corresponds to the energy threshold of photopion production of the highest energy cosmic-ray proton with the well measured cosmic microwave background photon field. Additionally, the neutrons created in the photopion interactions decay into electron anti-neutrinos with energies around a few PeV. Some models predict an additional contribution in the lower energy region around one PeV [5]. This contribution is highly dependent on higher energy (infrared, optical, and ultraviolet) background photons, as well as the transition energy between Galactic and extragalactic cosmic-rays. Above $\sim 1 \mathrm{EeV}\left(=10^{9} \mathrm{GeV}\right)$, the spectrum is expected to soften to $\sim E^{-2.0}$ until $\sim 100 \mathrm{EeV}$ where the spectrum becomes even steeper depending on the assumed maximal energies of cosmic-ray spectra at the production site. 


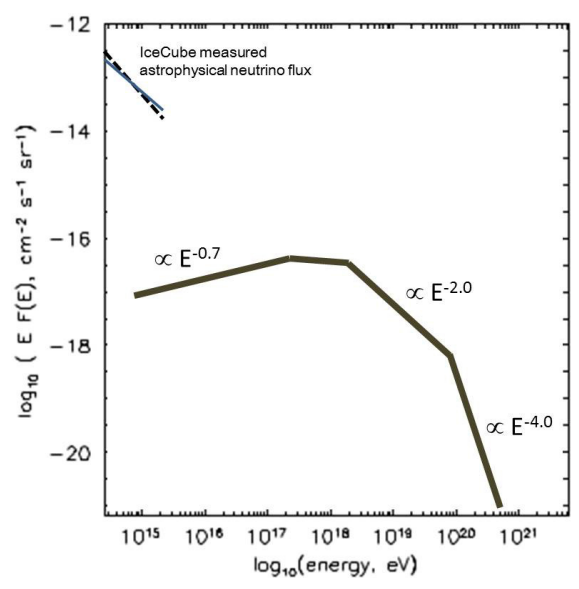

Figure 1: Expected cosmogenic neutrino spectrum and astrophysical neutrino spectrum measured by IceCube

As we see in the next sections, the effective detection area, or the exposure of the current analysis, scales approximately linearly with energy below EeV. The cosmogenic neutrino events observable by IceCube are expected to dominate in the energy range below a few EeV, above which a much more soft spectrum is expected.

When UHECRs interact with the photon field, only $v_{e}$ and $v_{\mu}$ are produced. Due to flavor oscillation, it is expected to become approximately $v_{e}: v_{\mu}: v_{\tau}=1: 1: 1$ at Earth. IceCube is sensitive to these three flavors of neutrinos. The largest contribution in the detection of high energy neutrino induced events above a PeV is from the "through-going muon" channel in which muons are created by $v_{\mu}$ charged current (CC) interactions outside the IceCube fiducial volume and then propagate through the detector emitting Cherenkov light from stochastic energy losses. Since average muons propagate more than $10 \mathrm{~km}$ above $\sim 1 \mathrm{PeV}$, this makes the sensitivity of IceCube extend to neutrino interactions far outside the IceCube detector volume. Similarly the contribution from "throughgoing tau" tracks from $v_{\tau} \mathrm{CC}$ interactions is important in the energy range where the GZK neutrinos are expected, since the tau decay length becomes more than a few $\mathrm{km}$ for $\geq 10^{8} \mathrm{GeV}$ whereas it is a few tens of meters for PeV $\tau$ s. In addition, particle showers produced at the neutrino interaction vertex position are observed as "cascade" events. Cascade events are induced by neutral current (NC) interactions by all three flavors of neutrinos as well as $v_{e} \mathrm{CC}$ interactions. While the cascade channel is limited to the interactions occurring inside or near the IceCube detector, their energy deposits in the detector are typically larger than that of muons or taus with equal energy. There is also an interesting contribution from the Glashow resonance [6] at $6.3 \mathrm{PeV}$, which results when an anti-electron neutrino interacts with an electron in the Earth. This interaction will result in an electron, tau, or hadron induced particle shower, or a muon track.

At energies above $\sim 1 \mathrm{PeV}$, the Earth is not transparent to neutrinos as the neutrino-nucleon cross section increases with energy. Thus, extremely high energy (EHE) neutrinos above the PeVscale, and their charged secondaries, are able to reach IceCube only from above and slightly below the horizon. Furthermore, the topological features of neutrino induced events change with energy even in each detection channel. For instance, neutrino induced muons exhibit more and more stochastic in energy losses at high energies. In addition, neutrino induced tau events drastically 
change from spherical to track-like shapes with increasing energy and above a few PeV electron induced particle showers become elongated due to the LPM effect [7].

\section{Signal selections}

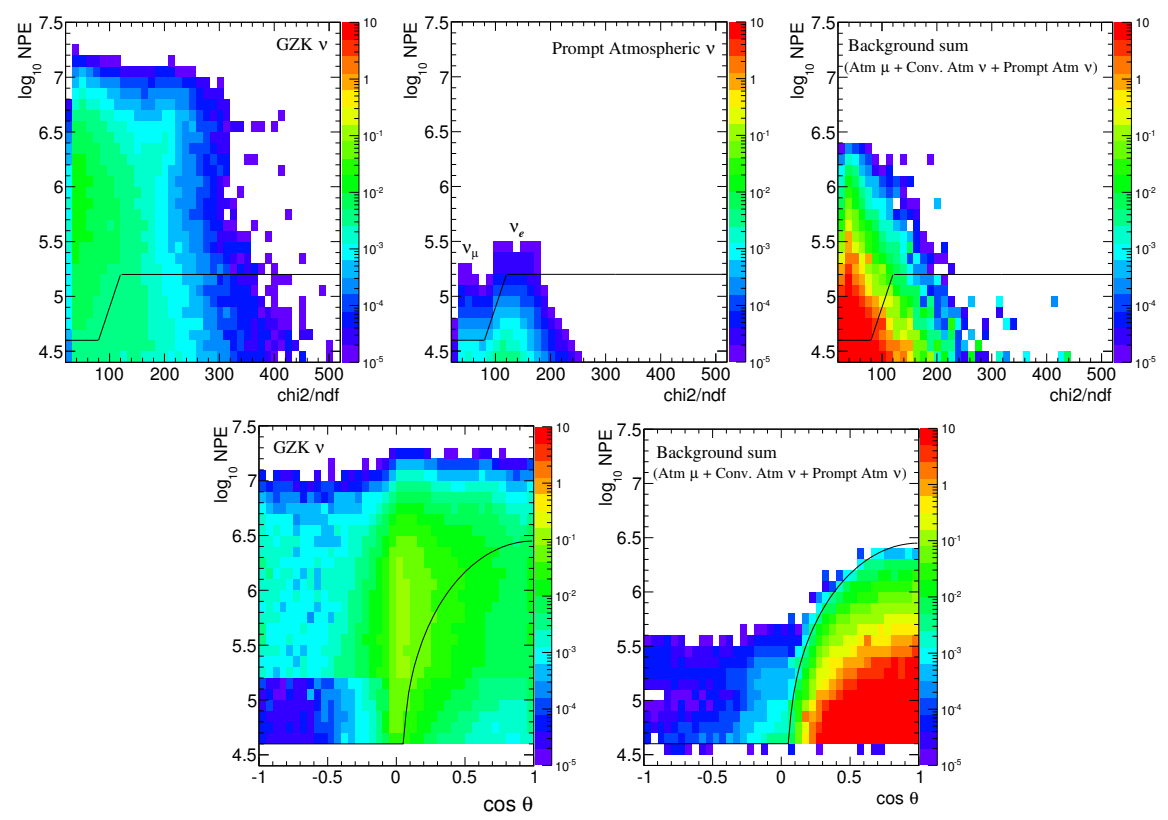

Figure 2: Event number distributions before Level-3 cut (upper panels) and Level-4 cut (lower panels) of the sample corresponding to 2050 days of livetime. The signal distributions in the left panels are from [13] adding all three flavors of neutrinos. The solid lines in each panel indicate the selection criteria in which only the events above the lines are kept. Event distributions from different detector configurations are added. The selection criteria are constant for the samples taken in different data taking periods. Atmospheric muons in the total background shown in the right panels are simulated with pure iron primary cosmic rays. In the case of pure proton primary, the background events are estimated to be a factor of 8 reduced. The upper middle plot shows the prompt atmospheric neutrino distribution to be removed by the Level-3 cut for reference.

The primary background in this analysis is downward-going muon bundles made up of large numbers of muons produced by high energy cosmic-ray interactions in the atmosphere. The secondary background is atmospheric neutrinos. Atmospheric neutrinos are induced by decays of charged mesons produced in cosmic ray interactions with the atmosphere. In particular in the energy region under study, prompt atmospheric neutrinos from decays of short lived heavy mesons are considered to dominate over conventional atmospheric neutrinos from pion and kaon decay. Unlike conventional atmospheric neutrinos, which are dominated by $v_{\mu}$, prompt neutrinos are composed of approximately equal fluxes of $v_{\mu}$ and $v_{e}$. While there must be non-zero prompt atmospheric neutrinos, no experimental observation of a prompt neutrino flux has been made, thus still relatively large theoretical uncertainty remains. In the current study, the model from [8] is considered as the default model. In this analysis, atmospheric muons were simulated with the CORSIKA air-shower simulation package version 6.720 [9] with the SIBYLL 2.1 [10] hadronic interaction model assuming pure proton and iron primary compositions in the energy region between $10^{5}$ and $10^{11} \mathrm{GeV}$. EHE 
neutrino signal events with energy between $10^{5}$ and $10^{11} \mathrm{GeV}$ were simulated using the JULIeT package [11].

In the current analysis, signal candidate events are extracted by applying four consecutive background rejection criteria. A large amount of low energy atmospheric muon induced events, typically less than a few $\mathrm{TeV}$, are discriminated by requiring the total number of photo-electrons (NPE) to be greater than 30,000 photo-electrons (p.e.) in the "Level-2" sample. This Level-2 data is subject to the "Level-3" cut which aims to remove atmospheric neutrinos, in particular the prompt neutrinos with a large theoretical uncertainty and atmospheric muons whose direction is not reliably reconstructed. The "Level-4" criterion is optimized to remove well reconstructed atmospheric muons of downward-going directions. Then the final "Level-5" criteria assure that there are no IceTop hits from cosmic-ray air-showers associated with the near final level events.

Figure 2 shows the event distributions as function of $\chi^{2} /$ ndf value and the total number of photo-electrons (NPE) as well as a function of $\cos \theta$ and NPE. The Level-3 and Level-4 NPE threshold as function of reduced $\chi^{2}$ and cosine of zenith angle are shown as solid lines in Fig. 2. In the Level-4 cut, the NPE threshold for the vertically downward going region is set to a high value $\left(\sim 2 \times 10^{6}\right)$ to reject energetic high multiplicity muon bundle events induced by ultra-high-energy cosmic rays. The NPE threshold is gradually reduced towards the horizontal direction because the number and energy of penetrating background atmospheric muons is significantly reduced for the inclined events. An NPE threshold value of $\sim 4 \times 10^{4}$ is assigned for the events near and below the horizon such that signal EHE cosmogenic neutrino induced events near the horizon can be selected efficiently while rejecting the high energy muon events dominant in the downward-going region and atmospheric neutrinos down to the level of 0.069 events per approximately 6 years of livetime. The Level-4 background events include 0.017 events of atmospheric muons, 0.019 events of conventional atmospheric neutrinos, and 0.033 events of prompt atmospheric neutrinos. To find correlated IceTop hits in the final level cut, downward-going reconstructed event tracks in the ice are extrapolated to the ice surface. Then the time $t_{C A}$, when the extrapolated tracks are at the closest approach to IceTop tanks, is calculated. The number of the correlated IceTop hits is defined by the number of IceTop hits in the time interval of $-1 \mu \mathrm{sec} \leq t_{C A} \leq 1.5 \mu \mathrm{sec}$. The final level selection requires the number of the correlated IceTop hits of the event to be less than two. A similar, but more detailed, analysis using the IceTop hits can be found in these proceedings [12].

\section{Expected performance}

The quasi-differential, model-independent sensitivity of the IceCube detector at 90\% CL per energy decade on neutrino fluxes above $10^{15} \mathrm{eV}(1 \mathrm{PeV})$ is shown in Fig. 3 assuming full standard neutrino flavor mixing. The corresponding sensitivity for a diffuse flux of cosmic neutrinos with an $E^{-2}$ spectrum in the central $90 \%$ energy range from $300 \mathrm{TeV}$ to $2 \mathrm{EeV}$ is calculated to be $E^{2} \phi \leq 3 \times 10^{-9} \mathrm{GeVcm}^{-2} \mathrm{sec}^{-1} \mathrm{sr}^{-1}$ with the 6 years of observation. The sensitivity improved by a factor of 2.5 from the previous analysis with approximately two years of IceCube data [4]. Table 1 gives the event rates for several model fluxes for cosmogenic neutrinos assuming the highest energy cosmic-rays above $5 \times 10^{19} \mathrm{eV}$ are protons only. In the present study, at most $\sim 5$ cosmogenic neutrino events from the models which have not been excluded are expected. This reflects the fact 


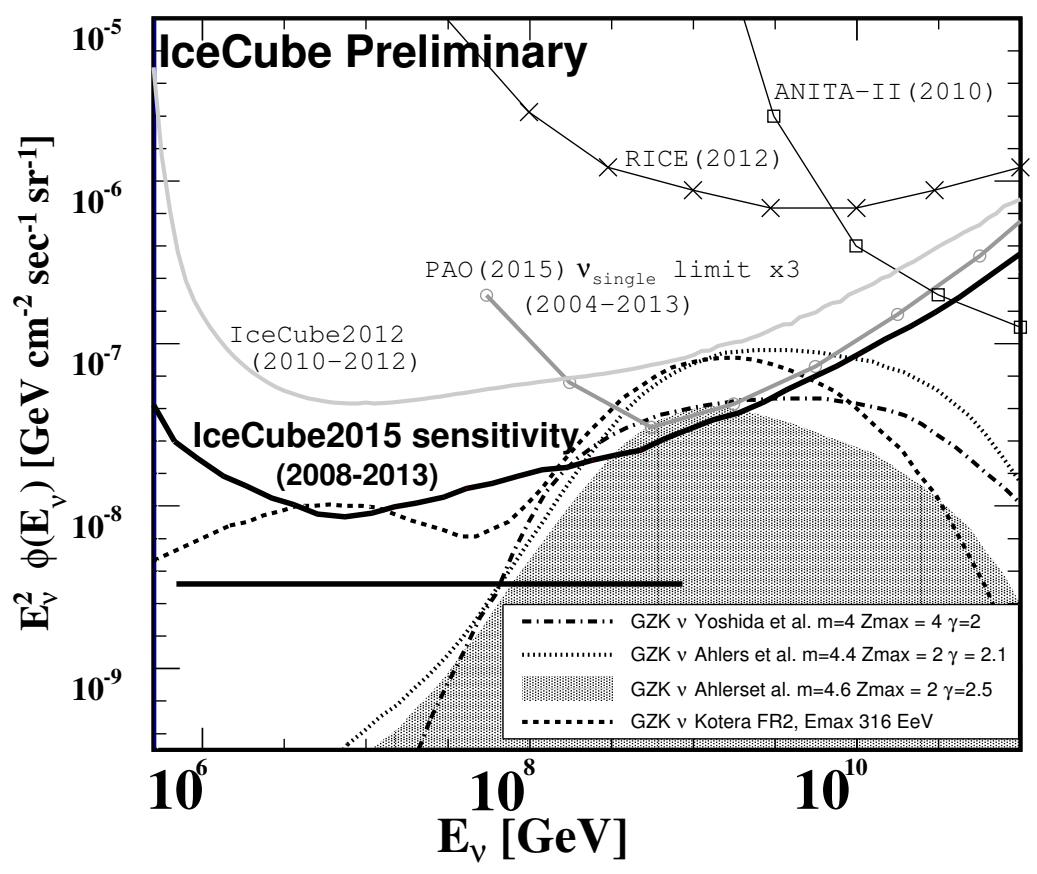

Figure 3: All flavor neutrino flux differential sensitivities of the IceCube detector. Several model predictions (assuming primary protons) are shown for comparison: Ahlers-max (the maximal fit), Ahlers-best (the best fit, incorporating the Fermi-LAT bound) [13], and Kotera-SFR [5] Yoshida- $(4,4)\left(\left(\mathrm{m}, \mathrm{Z}_{\max }\right)=(4,4)\right)[14]$. Model fluxes are summed over all neutrino flavors, assuming standard neutrino oscillations. The model independent differential upper limits by other experiments are also shown for Auger (PAO) [15], RICE [16], ANITA-II [17] Limits from other experiments are converted to the all flavor limit assuming standard neutrino oscillation and a 90\% quasi-differential limit per one energy decade when necessary. Errors not included.

that the number of expected signal events is increased by a factor of $\sim 2$ from the previous study, while a similar background event rate of 0.069 is expected in the total livetime.

The corresponding neutrino effective area averaged over $4 \pi$ solid angle is shown in the upper plots in Fig. 4. The neutrino effective area represents the surface area of an equivalent detector if it were $100 \%$ efficient, and it is proportional to the exposure. The lower panels in Fig. 4 show the Level-4 signal event distributions of each neutrino flavor as a function of neutrino energy at the Earth's surface. It can be seen that the present analysis is sensitive to all three neutrino flavors while the contribution of $v_{e}$ is larger near the threshold and decreases with energy.

\section{Summary}

The selection criteria for the 6 years of IceCube with different detector configurations and the associated analysis sensitivity is presented. The improvement in sensitivity from the previous twoyear analysis is approximately a factor of 2.5 . IceCube has proven to be the most powerful detector for extraterrestrial neutrinos between several $\mathrm{TeV}$ and several $\mathrm{PeV}$. The performance of IceCube in the highest energy region is also expected to be sufficient for the first detection of cosmological neutrinos if the highest energy cosmic-rays have a proton composition. The anticipation for this first detection is thus eagerly awaited. 

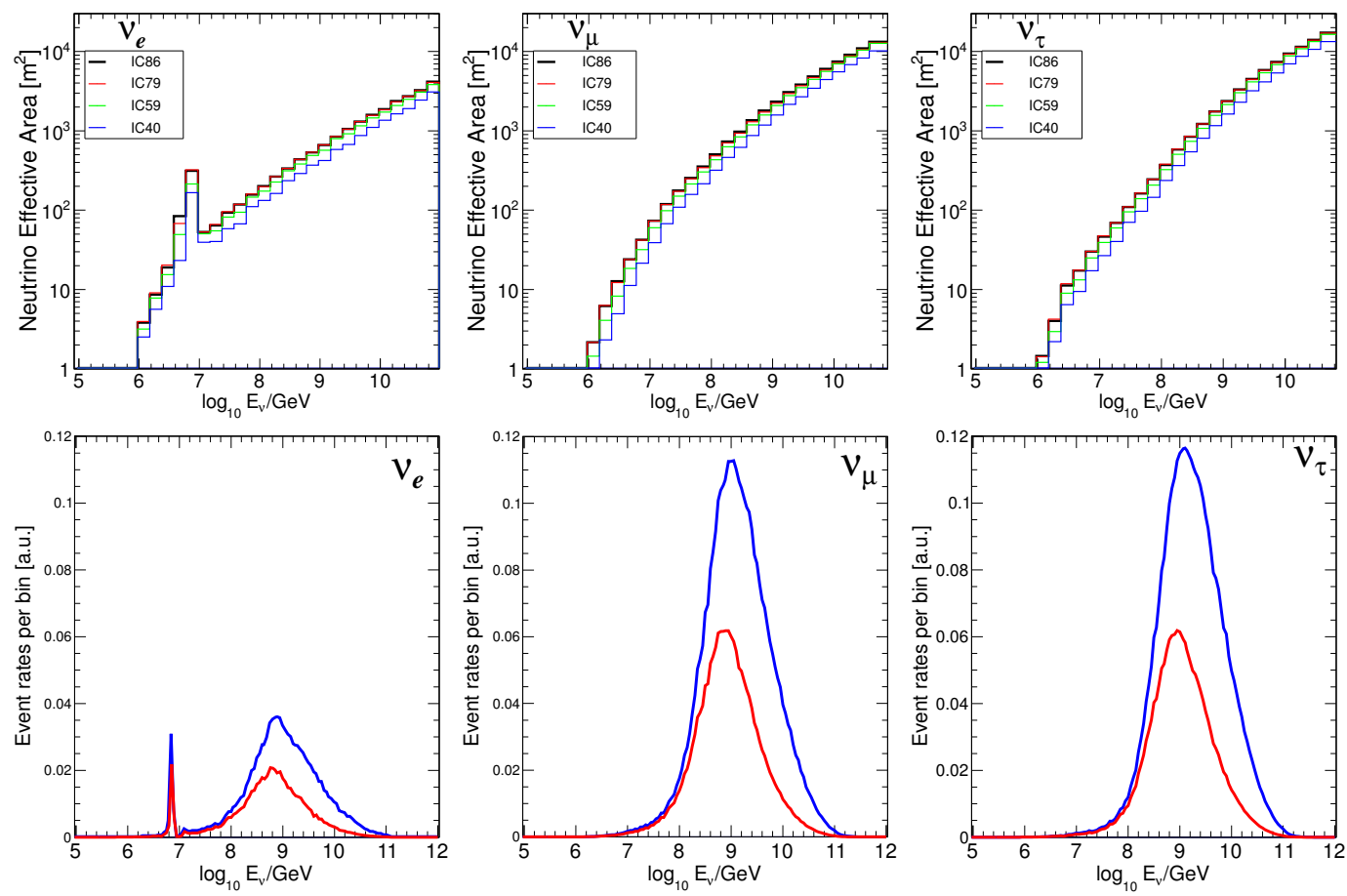

Figure 4: Upper panels show $4 \pi$ solid angle averaged neutrino effective area for each neutrino flavor and IceCube detector configuration. Each line represented by IC40, IC59, IC79 and IC86 corresponds to effective area for 40, 59, 79 and 86 (full) arrays, respectively. The lower panels present the expected event distribution as function of neutrino energy which passes the Level-4 criteria assuming Ahlers-Max (blue line) and AhlersBest (red line) model from [13].

\begin{tabular}{|c|c|c|c|c||c|}
\hline & \multicolumn{4}{|c||}{ model parameters } & Event rates in 2050 days \\
IceCube 2008-2014 \\
\hline Models & $\mathrm{m}$ & $Z_{\max }$ & $\gamma$ & $E_{\max }$ & $7.81 \pm 0.03$ \\
\hline \hline Ahlers-Max [13] & 4.4 & 2 & 2.1 & $10^{21} \mathrm{eV}$ & $3.87 \pm 0.02$ \\
Ahlers-Best [13] & 4.6 & 2 & 2.5 & $10^{21} \mathrm{eV}$ & $5.08 \pm 0.02$ \\
Yoshida-(4,4) [14] & 4 & 4 & 2.0 & $10^{22} \mathrm{eV}$ & $0.76 \pm 0.01$ \\
Yoshida-(2,4) [14] & 2 & 4 & 2.0 & $10^{22} \mathrm{eV}$ & $2.02 \pm 0.04$ \\
Kotera-GRB [5] & GRB & GRB & 2.0 & $3 \times 10^{20} \mathrm{eV}$ & $2.60 \pm 0.07$ \\
Kotera-SFR [5] & SFR & SFR & 2.0 & $3 \times 10^{20} \mathrm{eV}$ & \\
\hline
\end{tabular}

Table 1: Expected number of events detected by IceCube in 6 years from several cosmogenic neutrino models assuming the cosmic-ray primaries to be protons. The spectral indices $\gamma$, cutoff energies $E_{\max }$ at sources as well as cosmological evolution indices $m$ and extensions in redshift $Z_{\max }$ for the cosmic-ray sources are also listed for reference. The corresponding expected number of background events in one year is $0.069 \pm 0.003$. Errors are statistical only. 


\section{References}

[1] V. S. Berezinsky and G. T. Zatsepin, Phys. Lett. 28B, 423 (1969).

[2] K. Greisen, Phys. Rev. Lett. 16, 748 (1966); G. T. Zatsepin and V. A. Kuzmin, Pisma Zh. Eksp. Teor. Fiz. 4, 114 (1966) [JETP. Lett. 4, 78 (1966)].

[3] M. G. Aartsen et al. (IceCube Collaboration), Phys. Rev. Lett. 111, 021103 (2013).

[4] M. G. Aartsen et al. (IceCube Collaboration), Phys. Rev. D 88, 112008 (2013).

[5] K. Kotera, D. Allard, and A. Olinto, J. Cosmol. Astropart. Phys. 10, 013 (2010).

[6] S.L. Glashow, Physical Review 118, 316 (1960).

[7] For a review, see: S. Klein, Review of Mod. Phys. 71, 1501 (1999).

[8] R. Enberg, M.H. Reno, and I. Sarcevic., Phys. Rev. D 78, 043005 (2008).

[9] D. Heck et al., Report FZKA 6019, (Forschungszentrum Karlsruhe 1998).

[10] E. J. Ahn, R. Engel, T. K. Gaisser, P. Lipari, and T. Stanev, Phys. Rev. D 80, 094003 (2009).

[11] S. Yoshida et al., Phys. Rev. D 69, 103004 (2004).

[12] D. Tosi and K. Jero for the IceCube Collaboration, PoS(ICRC2015)1086, these proceedings.

[13] M. Ahlers and F. Halzen, Phys. Rev. D 86, 083010 (2012).

[14] S. Yoshida and M. Teshima, Prog. Theor. Phys. 89, 833 (1993).

[15] A. Aab et al. (Pierre Auger Collaboration), Phys. Rev. D 91, 092008 (2015).

[16] I. Kravchenko et al. (RICE collaboration), Phys. Rev. D 85, 062004 (2012).

[17] P. W. Gorham et al. (ANITA Collaboration), Phys. Rev. D 82, 022004 (2010); P. W. Gorham et al. (ANITA Collaboration), Phys. Rev. D 85, 049901 (2012). 